\title{
Sub Part-Per-Million Mass Accuracy by Using Stepwise-External Calibration in Fourier Transform Ion Cyclotron Resonance Mass Spectrometry
}

\author{
Richard L. Wong and I. Jonathan Amster \\ Department of Chemistry, University of Georgia, Athens, Georgia, USA
}

\begin{abstract}
A new external calibration procedure for FT-ICR mass spectrometry is presented, stepwiseexternal calibration. This method is demonstrated for MALDI analysis of peptide mixtures, but is applicable to any ionization method. For this procedure, the masses of analyte peaks are first accurately measured at a low trapping potential $(0.63 \mathrm{~V})$ using external calibration. These accurately determined ( $<1 \mathrm{ppm}$ accuracy) analyte peaks are used as internal calibrant points for a second mass spectrum that is acquired for the same sample at a higher trapping potential $(1.0 \mathrm{~V})$. The second mass spectrum has a $\sim 10$-fold improvement in detection dynamic range compared with the first spectrum acquired at a low trapping potential. A calibration equation that accounts for local and global space charge is shown to provide mass accuracy with external calibration that is nearly identical to that of internal calibration, without the drawbacks of experimental complexity or reduction of abundance dynamic range. For the 609 mass peaks measured using stepwise-external calibration method, the root-mean-square error is $0.9 \mathrm{ppm}$. The errors appear to have a Gaussian distribution; $99.3 \%$ of the mass errors are shown to lie within three times the sample standard deviation $(2.6 \mathrm{ppm})$ of their true value. (J Am Soc Mass Spectrom 2006, 17, 1681-1691) (c) 2006 American Society for Mass Spectrometry
\end{abstract}

$\mathrm{T}$ The introduction of electrospray ionization (ESI) [1-3] and matrix-assisted laser desorption/ionization (MALDI) [4-6] has led to a rapid growth in the application of mass spectrometry to biological analysis. With the growing availability of complete genome sequences, the demand for proteome analysis has increased dramatically. Protein samples of biological origins are highly complex and require analytical tools that have high sensitivity, wide dynamic range, high throughput, and the ability for automation. Mass spectrometry is now widely recognized as a powerful tool for proteomics.

Although protein identification can be classified into many categories, such as "top-down" [7-9] versus "bottom-up" [10-13], and shotgun methods [12, 14-19] versus peptide mass fingerprinting [20-23], protein identification is ultimately based on the mass measurement of proteins, peptides, or their fragment ions. A greater confidence in the accuracy of the mass measurement can improve the identification rate and the confidence level of the assignments. Of all types of mass analyzers, Fourier-transform ion cyclotron resonance

Published online August 24, 2006

Address reprint requests to Dr. I. J. Amster, Department of Chemistry, University of Georgia, Athens, GA 30602-2556, USA. E-mail: jamster@ uga.edu
(FT-ICR) mass spectrometry, developed by Comisarow and Marshall [24, 25], provides the highest mass accuracy over a broad $\mathrm{m} / \mathrm{z}$ range $[26,27]$ and the highest mass resolution [28], making identification of peptide elemental composition possible [29, 30]. Although sub part-per-million (ppm) mass accuracy can be achieved by FT-ICR [26, 27, 31], the typical accuracy level is usually in the 1 to $10 \mathrm{ppm}$ range. For external calibration, the mass accuracy in a FT-ICR experiment depends on the number of ions in the analyzer cell because a space-charge frequency shift causes the observed cyclotron frequency to decrease with increasing ion population [32-39]. Analyte separation before mass spectrometry is often necessary for proteome samples to reduce the sample complexity and to improve the detection dynamic range. However, the analyte ion production varies widely in liquid chromatographymass spectrometry (LC-MS) experiments, and the ion population in the analyzer cell can fluctuate by two to three orders of magnitude [40-43], resulting in systematic mass measurement offsets. In fact, greater abundance dynamic range for proteomics can be achieved by increasing the separation power before mass spectrometry, but at the expense of greater fluctuations in the resulting ion population.

Ultrahigh mass accuracy in FT-ICR can be achieved by using internal calibration with a small ion popula- 
tion, where space-charge frequency shifts can be treated with relatively simple equations [33, 34]. Even though the average mass error is minimized in internal calibration experiments, the ion population needs to be kept low to reduce the data scattering [44], but this condition produces spectra of poor sensitivity and poor abundance dynamic range, in opposition to the essential demands of a proteome analysis. To accommodate higher ion populations, Eyler and coworkers [31] and Smith and coworkers [44] have incorporated ion intensity as part of the fitting parameters for the calibration equation developed by Gross and coworkers [34]:

$$
\left(\frac{m}{z}\right)_{i}=\frac{A}{f_{i}}+\frac{B}{f_{i}^{2}}+C \cdot \frac{I_{i}}{f_{i}^{2}}
$$

where $f_{i}$ is the measured cyclotron frequency for a calibrant ion at $(\mathrm{m} / \mathrm{z})_{i}, I_{i}$ is the corresponding ion intensity, and $A, B, C$ are the regression fitting parameters. $A$ accounts for the magnetic field effect, $B$ and $C$ terms account for the global and local space-charge effects, respectively. The space-charge frequency shift caused by ions of the same $m / z$ (local space-charge) is treated separately from the rest of the space-charge frequency shift (global space-charge) when used with internal calibration. This modified calibration equation has been shown to improve internal calibration mass accuracy by a factor of 1.5 to 6.7 , depending on the calibration mass range and the ion excitation radius [44]. The new calibration equation is especially useful for proteomic studies where a high ion population in the analyzer cell is essential to achieve a high dynamic range in the abundance scale. However, internal calibration for complex mixtures usually requires a specialized instrument setup, such as a dual-ESI ionization source $[45,46]$ or the means to accumulate ions desorbed from multiple MALDI sample spots [38, 47]. A high level of skill is required to properly implement such devices and, thus, these techniques have not been widely adopted. Moreover, adding calibrant ions to the analyzer cell complicates the resulting mass spectrum and raises the likelihood of mass overlap between analyte and calibrant species. Furthermore, it decreases the detection dynamic range by using some of the available charge capacity of the analyzer cell for nonanalyte ions.

For external calibration, the space-charge effects on mass accuracy can be reduced by using a calibration curve of frequency shift versus ion population as demonstrated by McIver and coworkers [33] and Amster and coworkers [37, 39]. Muddiman and Oberg [48] applied a global regression calibration approach by separately treating the total ion intensity from intensity of the ions of interest, and achieved a mass accuracy of $<5 \mathrm{ppm}$ using external calibration for polypropylene glycol. Nonetheless, making an accurate frequency shift/ion abundance curve can be time-consuming and the calibration curve is only suitable for a single set of experimental conditions. Another approach for external calibration is to precisely control the ion population in the analyzer cell using automatic gain control (AGC) [49]. Such an approach has been implemented on a commercial ESI-FTICR device and is claimed to routinely produce mass errors of less than 2 ppm $[49,50]$. However, Smith and coworkers demonstrated that mass accuracy using AGC depends strongly on the selected abundance level of the ion population. The mass accuracy obtained with a high ion population in the analyzer cell is not as good as for a low population [44, 51]. The mass confidence levels using AGC reported by the Gygi and Smith groups are $\sim 5 \mathrm{ppm}$ for external calibration experiments [18, 46, 51]. While AGC improves mass accuracy for ESI-FTICR experiments, the implementation is not suitable for pulsed ion sources. Other approaches are needed for attaining high mass accuracy in MALDI experiments.

Here, we describe a two-step calibration procedure for FT-ICR, which can be readily applied to any complex analyte, that requires no specialized hardware such as is required for AGC, and which can be used for MALDI or ESI experiments. The analyte mass spectrum is first acquired using external calibration at a low trapping potential $(0.63 \mathrm{~V})$, which provides high mass accuracy but low dynamic range for ion abundance. A second analyte mass spectrum is then acquired at a higher trapping potential $(1.0 \mathrm{~V})$, which significantly improves signal-to-noise and the dynamic range for abundance measurements. The mass values measured at the low trapping potential are used as calibration reference points for the second spectrum. This stepwiseexternal calibration method is tested on three different protein digest systems and compared to other calibration methods. Moreover, a new calibration equation that corrects for local space-charge is incorporated in the stepwise-external calibration approach and investigated. Stepwise-external calibration provides comparable mass accuracy to internal calibration without its experimental complexity or the other above-mentioned shortcomings.

\section{Experimental}

\section{Materials and Sample Preparation}

2,5-Dihydroxybenzoic acid (DHB) and dithiothreitol (DTT) were purchased from Lancaster (Pelham, NH) and Sigma (St. Louis, MO), respectively. Trypsin, bovine serum albumin (BSA), and chicken egg albumin (ovalbumin) were purchased from Promega (Madison, WI), Sigma (St. Louis, MO), and Calbiochem (San Diego, CA), respectively. Protein samples were prepared at $\sim 1 \mathrm{mg} / \mathrm{mL}$ concentration and denatured by heating at $90^{\circ} \mathrm{C}$ for 5 to $10 \mathrm{~min}$. Disulfide bonds were reduced using $5 \mathrm{mM}$ DTT at $70^{\circ} \mathrm{C}$ for $1 \mathrm{~h}$. Denatured proteins were digested overnight at $37^{\circ} \mathrm{C}$ using trypsin at a 1:50 protease:protein ratio (by mass). Four hundred $\mathrm{nL}$ of the digested proteins was applied to a stainless steel matrix-assisted laser desorption/ionization (MALDI) plate and $400 \mathrm{~nL}$ of $1 \mathrm{M}$ DHB prepared in 
50:50:0.1\% water:acetonitrile:trifluoroacetic acid solution (by volume) was added as the MALDI matrix.

\section{Mass Spectrometry}

Mass spectra were collected on a 9.4 tesla Bruker (Billerica, MA) BioApex Fourier-transform ion cyclotron resonance (FT-ICR) mass spectrometer equipped with an intermediate pressure Scout 100 MALDI source. Ions generated from five MALDI laser shots were accumulated in a hexapole. Argon gas was pulsed into the source region during MALDI events to enhance ion accumulation in the hexapole and to reduce the kinetic and internal energy of the ions. The accumulated ions were released from the hexapole by reducing the voltage applied to the hexapole exit electrode, and were guided to the FT-ICR analyzer cell through a series of electrostatic ion optics. The mass range of the detected ions can be selected by varying the ion extraction time, that is, the period between the ejection of ions from the source hexapole and the beginning of ion excitation and detection [19]. For acquisition of the BSA tryptic digest mass spectra, data were collected with ion extraction times of 2 and $4 \mathrm{~ms}$ to enhance the low and high mass ions, respectively. For acquisition of the ovalbumin digest mass spectra, data were collected using an ion extraction time of $4 \mathrm{~ms}$ only. Ions were excited using a chirp waveform (125 steps, $2 \mathrm{kHz} / \mathrm{step}, 0.32 \mu \mathrm{s} / \mathrm{step}, 40$ $\mu$ s total sweep time, sweep range $36088 \mathrm{~Hz}-294117 \mathrm{~Hz}$, $400 \mathrm{~V}_{\mathrm{p}-\mathrm{p}}$ ) and $1 \mathrm{M}$ point transients were acquired at an analog-to-digital conversion rate of $588 \mathrm{kHz}$. The data were apodized with a sinebell function and padded with one zero-fill before fast Fourier transformation and magnitude calculation to the frequency domain. The mass spectra collected using the above conditions have a lower mass limit of $\mathrm{m} / \mathrm{z}$ 490. A few spectra were acquired using a lower mass limit of $m / z 100$ to ensure that matrix ions or other lower mass species were not transferred to the analyzer cell. Spot-to-spot variation in the MALDI process was used to generate mass spectra with a wide range of total ion intensities.

\section{Stepwise-External Calibration}

For stepwise external calibration, a mass spectrum is acquired at a trapping potential of $0.65 \mathrm{~V}$. This mass spectrum is externally calibrated using the standard formula, eq 2. For the present work, the two calibration constants are obtained from a mass spectrum of a mixture of peptides of known composition from a protein proteolytic digest, but any mass standards are suitable, provided that the acquisition parameters are identical for the mass spectra of calibrant and the sample. A second mass spectrum of the same sample is next acquired at a trapping potential of $1.0 \mathrm{~V}$. Generally, all of the peaks in the mass spectrum obtained in the first mass spectrum (0.65 V trapping potential) will appear in the second mass spectrum, and will have higher abundance than in the first mass spectrum, allowing one to easily correlate the peaks from the two mass spectra. In addition, many new low abundance peaks will appear in the second mass spectrum. An internal calibration is applied to the second mass spectrum, using the masses that were measured in the first mass spectrum. In the present work, from 12 to 15 peaks were selected as internal calibrants, leaving the remainder of the assignable peaks for testing the mass accuracy of the calibration method. In practice, all of the peaks in the first mass spectrum would be used as internal calibrants, as the resulting mass errors are reduced as the number of calibrant peaks increases (vide infra). It is particularly useful to include both high and low abundance peaks when using eq 3 as the calibration formula, as this equation incorporates the intensity of a peak to account for local space charge effects. For internal calibration, linear regression is used to obtain the calibration constants by fitting the measured frequencies and intensities to the masses that are determined from the first mass spectrum, using the Microsoft Excel LINEST function.

\section{Results and Discussion}

To investigate the reliability of various calibration methods, tryptic peptides of bovine serum albumin (BSA) and chicken egg albumin (ovalbumin) are studied using MALDI-FTICR. By changing the time delay between ion introduction and cell accumulation, the range of masses that are trapped can be selected [19]. Tryptic fragments of BSA are detected by using low and high mass selective enhancement, as shown in Figure 1a and $b$, while ovalbumin fragments are detected using the high mass selective enhancing condition. For clarity of discussion, BSA mass spectra generated using the low and high mass enhancing conditions are denoted as BSALow and BSAHigh, respectively. The mass peaks marked with numerical values and open circles in Figure 1 correspond to predicted tryptic peptides. Peaks marked with their nominal mass values are used for calibrant points and for mass accuracy assessment, and those marked with open circles are treated as analyte peaks to test the mass accuracy. Twenty-one mass spectra are collected for BSALow, BSAHigh, and ovalbumin at $1.0 \mathrm{~V}$ and $0.63 \mathrm{~V}$ cell trapping potential-a total of 126 spectra. Unless specified, mass spectra discussed are acquired at a $1.0 \mathrm{~V}$ trapping potential.

Stepwise-external calibration is based on the observation that the best mass accuracy for FT-ICR is obtained when the trapping potential and ion population are low. By using low trapping potentials, the ion capacity of the cell is reduced significantly, so that a low population of ions is obtained even for high sample concentrations. By capping the upper limit of ion abundance, space charge induced frequency shifts are significantly reduced. Highly accurate mass values can be obtained using external calibration at a low trapping potential $(0.63 \mathrm{~V}$ for this experiment), but mass spectra obtained in this man- 


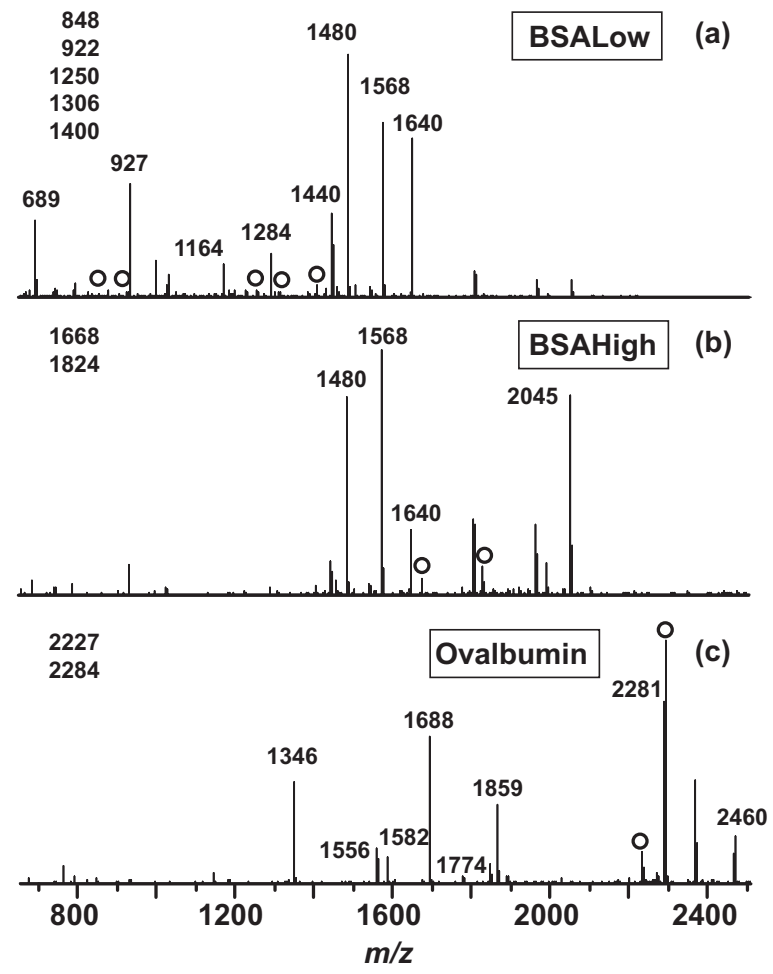

Figure 1. Mass spectra of (a) BSA tryptic digest acquired using low mass enhancing conditions, (b) BSA digest measured with high mass enhancing conditions, and (c) ovalbumin digest measured using high mass enhancing conditions. Peaks labeled with their nominal mass values are used for calibration points and for error assessment, whereas the peaks marked with open circles are only for error assessment, and their nominal mass values are listed on the top left of the spectra.

ner have reduced signal-to-noise and abundance dynamic range due to the smaller ion capacity of the analyzer cell. In addition, the relative abundances of the peaks are more susceptible to statistical fluctuation, and are less reliable for quantification. To recover the lost dynamic range and to maintain high mass accuracy, a new mass spectrum is acquired for the same sample at a higher trapping potential (1.0 $\mathrm{V})$, and the mass values measured using the low trapping potential, are used as calibration reference masses for the spectrum acquired at the higher trapping potential. This stepwise-external calibration mimics internal calibration via calibrating with mass peaks that lie within the analyte spectra. However, the peaks used for calibration are also analyte ions, and the reference mass values are obtained from a separately acquired spectrum using external calibration at a low trapping potential. In the present work, stepwise-external calibration is compared with conventional external calibration and internal calibration. In this study, the spectrum having the lowest total ion intensity within each category is used as the reference spectrum for conventional external calibration, to provide calibration parameters for the other spectra. Internal calibration is performed when spec- tra are calibrated on their known peaks, that is, the peaks labeled with numbers in Figure 1.

The accuracy of the stepwise-external calibration method largely depends on its first step: the ability to measure accurate mass values for the analyte at a low trapping potential via external calibration. To estimate the accuracy level of this step, 21 mass spectra are acquired using a low trapping potential $(0.63 \mathrm{~V})$ for each protein digest system. The spectrum having the lowest total ion intensity is used as the external calibration reference spectrum for the other 20 spectra, and the calibrated mass values of the highest ion intensity spectrum are used as the reference masses for spectra acquired at a higher trapping potential. This provides a "larger than average" space-charge effect for spectra measured at the low trapping potential and, therefore, tests the robustness of the stepwise-external calibration method.

To examine mass accuracy in a systematic fashion, the root-mean-square (RMS) of the errors, the average error (AVE), and the population standard deviation (S.D.) of the errors are calculated for each spectrum. The three terms are expressed as follows:

$$
\begin{aligned}
& R M S=\sqrt{\frac{\sum_{i}\left(\text { mass error }_{i}\right)^{2}}{n}} \\
& A V E=\frac{\sum_{i} \text { mass error } i}{n} \\
& S . D .=\sqrt{\frac{\sum_{i}\left(\text { mass error }_{i}-A V E\right)^{2}}{n}}
\end{aligned}
$$

where $i$ is the index number for mass peaks, $n$ is the total number of data, and mass error is expressed in parts-per-millions (ppm). The RMS error value indicates the accumulated error in a mass spectrum. The AVE error reflects the average position of the errors, allowing cancellation between positive and negative errors, while the S.D. value accounts for the discrepancy within the data. The population standard deviation expression is carefully chosen over the sample standard deviation because these S.D. values are not used for estimating confidence limit for the population. Instead, the S.D. values are used to represent the "nonaverage error". The three terms are directly related by the eq: $\mathrm{RMS}^{2}=\mathrm{AVE}^{2}+\mathrm{S} \cdot \mathrm{D}^{2}$ [52].

\section{Errors for the Calibrant Points in BSALow}

External calibration, internal calibration, and stepwiseexternal calibration methods were examined for BSALow at a $1.0 \mathrm{~V}$ trapping potential, which has 8 calibrant masses, ranging $\mathrm{m} / \mathrm{z} 689$ to 1640 (Figure 1a). The standard calibration equation used is developed by McIver and coworkers [33]: 
BSALow Errors for Global Space-Charge Correction via Equation 2 Calibrant Noncalibrant

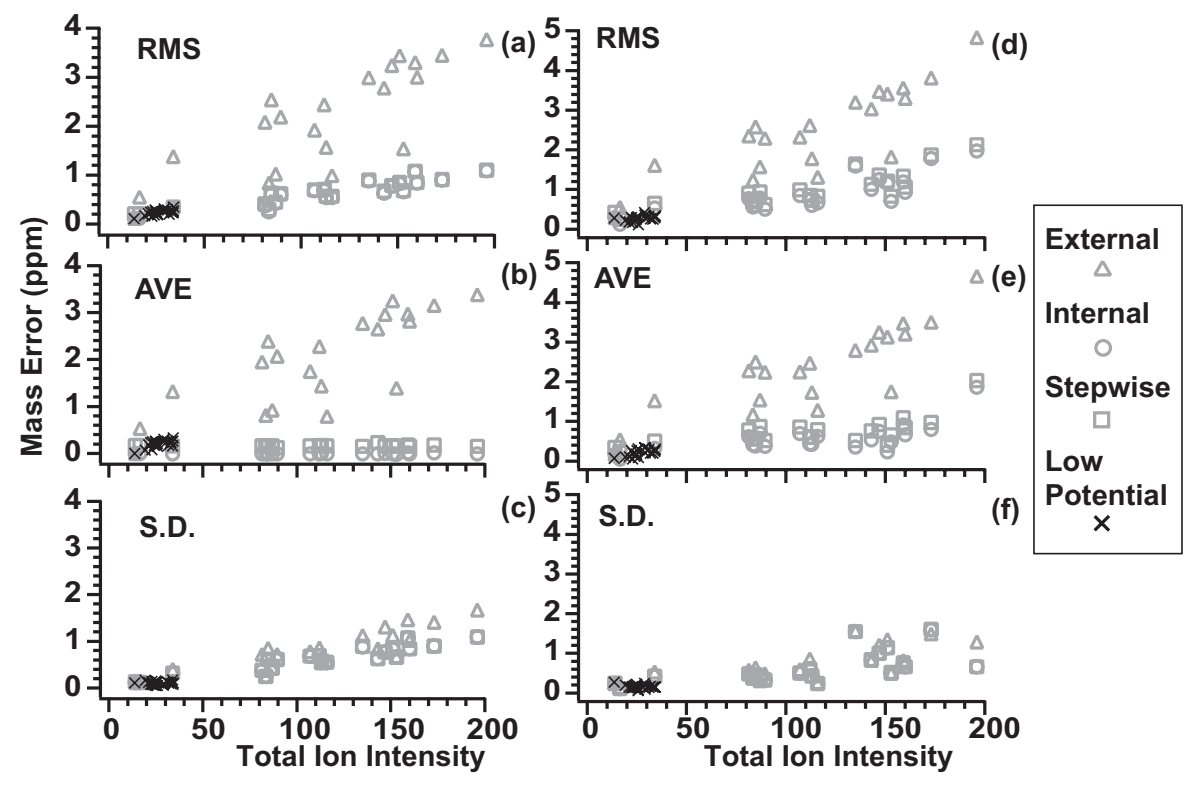

Figure 2. Error analysis for BSALow calibrant and noncalibrant masses accounting for global space-charge effects. (a) Root-mean-square error, (b) average error, (c) standard deviation of calibrant masses are plotted against total ion intensity for each spectrum, and (d) root-mean-square error, (e) average error, (f) standard deviation of noncalibrant masses are plotted against total ion intensity for each spectrum. Errors of external (triangles), internal (circles), and stepwise-external (squares) calibration are calculated from spectra acquired using a $1.0 \mathrm{~V}$ trapping potential, while the errors of low trapping potential external calibration (crosses) are calculated from spectra measured using a 0.63 $\mathrm{V}$ trapping potential.

$$
\frac{m}{z}=\frac{A}{f+B}
$$

where $f$ is the measured cyclotron frequency, $m / z$ is the mass-to-charge value, and $A$ and $B$ are fitting parameters. For internal calibration experiments, parameter $A$ of eq 2 accounts for the magnetic field, while $B$ accounts for the electric field from the trapping potential and from global space-charge effects $[32,33]$. The $B$ term is always positive because the electric field from the trapping potential or the global space-charge effect decreases the observed cyclotron frequency. Mass errors are calculated for the eight calibrant peaks for the various calibration methods. The RMS, AVE, and S.D. of the errors for each spectrum are plotted against the total ion intensity for the four calibration methods in Figure $2 \mathrm{a}, \mathrm{b}$, and c. The RMS error is largest with external calibration (triangles) and displays a strong dependence on the total ion intensity (Figure 2a), whereas the RMS errors for internal (circles) and stepwise-external (squares) calibration are essentially the same and have a much smaller dependence on the ion intensity. The external calibration obtained at a low trapping potential (crosses) spans a very narrow range of ion intensity and produces the smallest errors of the four methods. These results indicate that accurate masses are obtained via external calibration at a low trapping potential. The large RMS error for the external calibration data is largely due to global space-charge effects, where the measured cyclotron frequency for an ion decreases with increasing ion population in the analyzer cell. This effect is more clearly observed in the plot of AVE error versus total ion intensity, Figure 2b. The magnitudes of the RMS (triangles in Figure 2a) and AVE (triangles in Figure 2b) errors are similar for external calibration, indicating that a majority of the RMS error is due to AVE error, consistent with the constant error expected from the global space-charge effects. At the same time, the AVE errors for the internal (circles) and stepwise-external (squares) calibration are essentially independent of the ion intensity in Figure 2b, suggesting the two calibration methods are sufficient for minimizing the space-charge induced errors for these calibrant masses. The AVE error for stepwiseexternal calibration has a small constant offset because the calibrant mass values derived using external calibration at the low trapping potential also have a small offset.

The main source of mass errors for internal and stepwise-external calibration is the result of data scattering, as the RMS plots are similar to the S.D. plots for these two calibration methods (circles and squares in Figure 2a and c). The S.D. errors for the external, internal and stepwise-external calibration methods are very similar and show a small positive relationship with total ion intensity (Figure 2c), suggesting eq 2 
becomes less accurate for describing the mass-tofrequency relationship at high ion abundance.

\section{Local Space-Charge Effects on Mass Accuracy}

If local space-charge effects play a role in controlling mass accuracy [44], then standard calibration eq 2 will be insufficient for the prediction of mass values of peaks with large intensity differences from the calibrant peaks. To test this theory, five peaks with low intensity (circles) were selected from the mass spectrum shown in Figure 1a. The RMS errors for these noncalibrant peaks are shown in Figure 2d, and are seen to be larger than those of the calibrant points in Figure 2a. This is consistent with local space-charge effects that are unaccounted for via standard calibration eq 2 , however it could also result from the data regression procedure. Since calibration is performed using least-squares regression on the calibration reference points, the mass peaks that are directly calibrated generally have errors smaller than other peaks in the same mass spectrum. However, the AVE error plots in Figure 2e strongly suggest one must account for local space-charge effects to accurately measure the low abundance peaks. Not only are the AVE errors for internal (circles) and stepwise-external (squares) calibration in Figure 2e much greater than those in Figure $2 b$, but the AVE errors for the noncalibrant peaks actually increases with the total ion intensity of the spectra. One explanation for this observation is that space-charge forces are smaller between ions of the same $\mathrm{m} / \mathrm{z}$ than between ions of different $\mathrm{m} / \mathrm{z}$, resulting in a smaller space-charge frequency shift for the more intense calibrant peaks [44, 48]. Using the intense peaks for calibration reference points underestimates the frequency shift for the less intense ions. Consequently, the space-charge induced mass errors are only partially corrected in the case of the low abundance ions, resulting in a small dependence on ion abundance. The S.D. errors are similar for the three calibration methods suggesting that the data scattering is the same for three approaches (Figure 2f). The RMS, AVE, and S.D. errors are very small for external calibration data collected at a low trapping potential (crosses in Figure 2d, e, and f), where the range of ion intensities is small. These results again suggest the global and local space-charge effects are minimal at low ion abundance conditions, and thus the measured masses obtained at a low trapping potential serve as good reference masses.

To achieve better mass accuracy, we have tested calibration equations that account for local spacecharge effects. The two calibration approaches utilized are based on the modified calibration equation demonstrated by Eyler and coworkers [31] and Smith and coworkers [44], and a new implementation by Muddiman and Oberg [48]. In our study, the modified calibration equation, eq 3 , is an extension of the calibration eq 2 .

$$
\left(\frac{m}{z}\right)_{i}=\frac{A}{f_{i}+B+C \cdot I_{i}}
$$

where $I_{\mathrm{i}}$ is the intensity of an ion measured at frequency $f_{i}$ and has a mass of $(\mathrm{m} / \mathrm{z})_{i}$. As mentioned above, parameter $B$ corrects for the applied electric field (trapping potential) and global space-charge effects. Parameter $C$ acts as a correction factor for local space-charge effects. Although the expression of eq 3 differs from that of eq 1, the calibration results are similar. For the 21 spectra of BSALow, the internal calibration RMS errors for the calibrant peaks using eq 1 and eq 3 are $0.63 \mathrm{ppm}$ and 0.64 , respectively, and $0.93 \mathrm{ppm}$ and $0.91 \mathrm{ppm}$, respectively, for the noncalibrant points. The close agreement between the two forms of equation is expected. Marshall and coworkers have demonstrated that the two calibration equations developed by McIver and coworkers and Gross and coworkers produce essentially the same mass accuracy result [53]. For this calculation, the stepwise-external calibration approach is modified to mimic a more realistic situation by using additional detectable peaks (not noncalibrant peaks) collected at the low trapping potential as calibration reference masses. The identities of these peaks are inconclusive but they consistently appear in every spec-

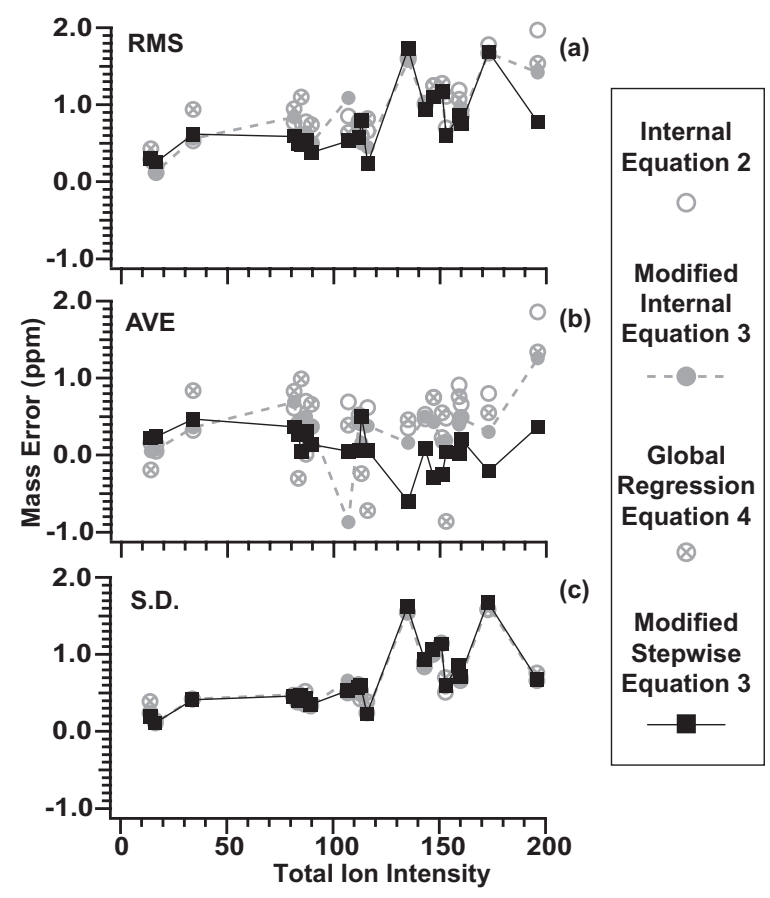

Figure 3. BSALow errors for noncalibrant masses; global and local space-charge correction. Improvement of mass errors for BSALow by accounting for local space-charge effects using eq 3 . (a) Root-mean-square error, (b) average error, and (c) standard deviation of noncalibrant masses for BSALow are plotted against total ion intensity for each spectrum. Errors of standard internal (open circles), modified internal (filled grey circles), modified global regression (circles with a cross), and modified stepwiseexternal (squares) calibration are calculated from spectra acquired using a $1.0 \mathrm{~V}$ trapping potential. 
trum. A main advantage of stepwise-external calibration over internal calibration is that no calibrant is added to the sample. Therefore, in a real stepwiseexternal calibration experiment, all detectable peaks obtained at a low trapping potential are equally good and are used as calibration reference masses. This modification provides stepwise-external calibration with more reference points over a wider intensity range.

The other approach is a global regression calibration method similar to that implemented by Muddiman and Oberg [48]. Instead of applying eq 3 to each individual spectrum, a global regression is preformed on all available spectra, in this case, the 21 spectra of BSALow. The global regression calibration equation is:

$$
\left(\frac{m}{z}\right)_{i}=\frac{A}{f_{i}+B+C \cdot I_{i}+D \cdot I_{\text {total }}}
$$

where $I_{\text {total }}$ is the sum of all ion intensity in a spectrum and $I_{i}$ is the intensity of the peak of interest measured with cyclotron frequency $f_{i}$.

Figure 3 shows the RMS, AVE, and S.D. errors of the noncalibrant points for internal calibration using standard calibration eq 2 (open circles) and modified eq 3 (filled circles), for global regression using eq 4 (circles with a cross) and for stepwise-external calibration using modified eq 3 (squares). The RMS errors are similar for the four methods, although the errors derived from standard calibration eq 2 (open circles) and global calibration eq 4 (circles with a cross) are marginally

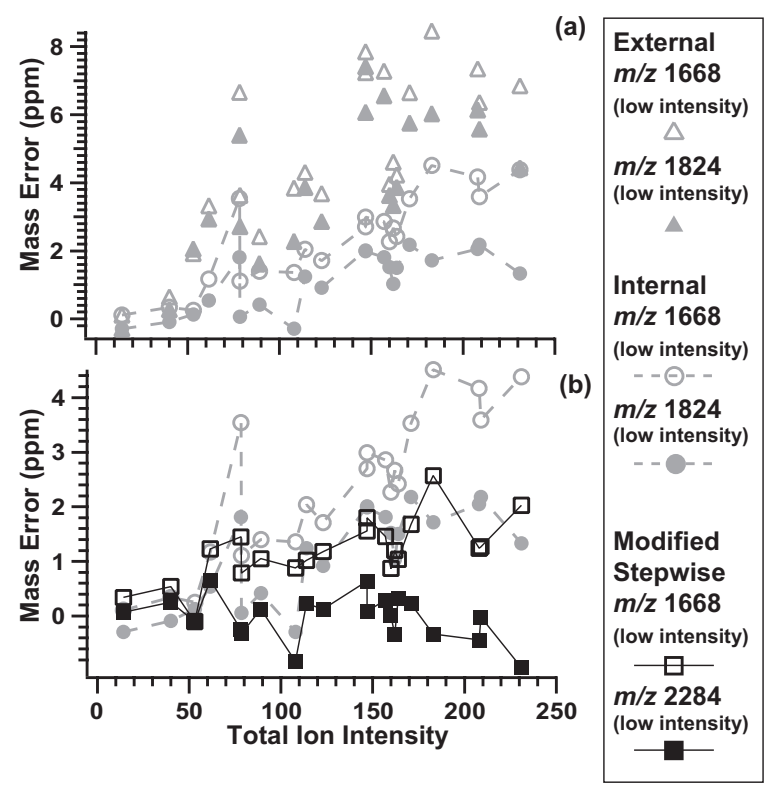

Figure 4. BSAHigh errors for noncalibrant masses. Error analysis comparison for two low intensity peaks. The mass errors of the noncalibrant peaks of $\mathrm{m} / \mathrm{z} 1668$ (open symbols) and 1824 (filled symbols) are plotted against total ion intensity for (a) comparing external calibration (triangles) to standard internal calibration (circles), and (b) comparing standard internal calibration (circles) with modified stepwise-external calibration (squares). larger. The improvement obtained by using the modified eq 3 is shown in Figure 3b, where the AVE errors are smaller for modified internal calibration (filled circles) and modified stepwise-external calibration (squares) comparing to the data derived from the standard internal calibration method (open circles). The low AVE error obtained for modified stepwise-external calibration shows that the systematic error associated with space-charge effects has been reduced to a fraction of the S.D. error of the measurement (Figure 3c). These results show that the space-charge frequency shifts of the low abundance peaks are properly accounted for by using modified eq 3 , even through they were not used as calibrant points.

A similar analysis is conducted for BSA fragments in which the heavier ions (m/z 1470 to 2050) shown in Figure $1 \mathrm{~b}$ are selectively trapped and detected. Because the noncalibrant peaks constitute the greater challenge, only their errors are discussed. As illustrated in Figure $1 b$, the ion intensities of the four calibrant peaks are noticeably higher than those of the two noncalibrant peaks ( $m / z 1668$ and 1824). In Figure 4a, the mass errors of the two noncalibrant peaks are individually plotted against total ion intensity for external calibration and internal calibration using standard calibration eq 2. The mass errors from external calibration (triangles) are reduced when internal calibration (circles) via eq 2 is used, but most of the mass errors are positive, indicating a systematic mass shift is still the main source of errors. The effect is due to space-charge effects that are not effectively corrected when the calibration is applied to the low abundance peaks. The mass errors are noticeably reduced when modified calibration eq 3 is used for stepwise-external calibration (squares) in Figure $4 \mathrm{~b}$. Much of this improvement is due to better treatment of the local space-charge effects for the stepwise-external approach. Similar to the AVE error of BSALow shown in Figure 3b, the mass errors of BSAHigh obtained using the modified stepwise-external calibration (squares) displays a smaller total ion intensity dependence than internal calibration using modified eq 3 (data not shown), illustrating the benefit of using additional calibrant points in stepwise-external calibration to offset the small error in the reference masses. To test this theory, errors were examined when the reference masses were limited to those used in the internal calibration for BSALow and BSAHigh. In these test cases, the modified stepwise-external calibration results became slightly worse than the modified internal calibration results, proving that the additional data points are beneficial. It is important to reiterate that in a typical complex spectrum of a complex mixture, stepwise-external calibration has the advantage of using any detectable peak at a low trapping potential for calibration and therefore spanning essentially the entire abundance dynamic range of the data. Nevertheless, mass error still increases with total ion intensity using the modified calibration. Since the average of mass errors has already been minimized for mass peaks with 


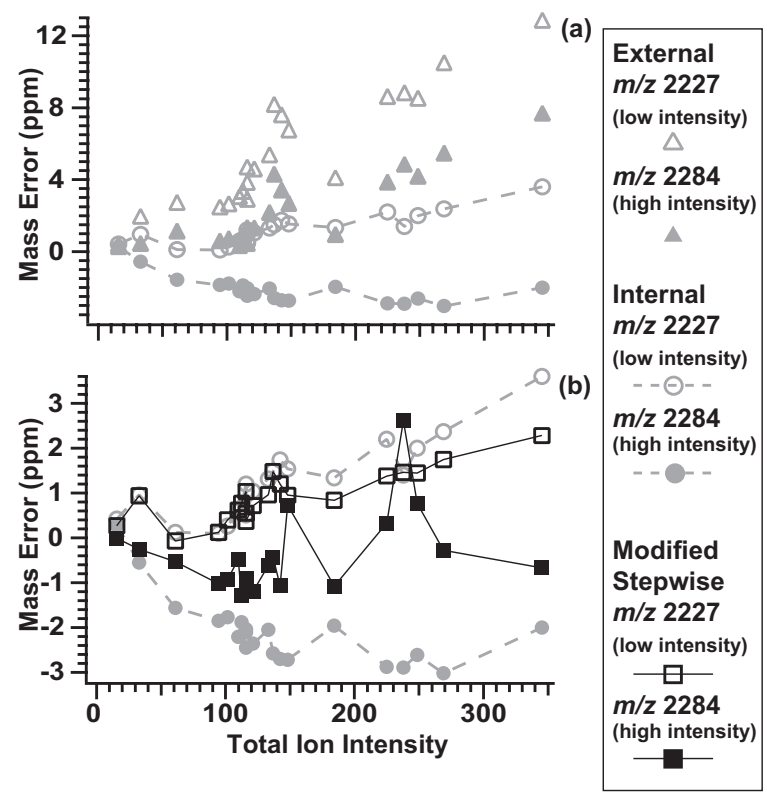

Figure 5. Ovalbumin errors for noncalibrant masses. Error analysis comparison for a low intensity versus a high intensity peak. The mass errors of the noncalibrant peaks of $\mathrm{m} / \mathrm{z} 2227$ (open symbols) and 2284 (filled symbols) are plotted against total ion intensity for (a) comparing external calibration (triangles) to standard internal calibration (circles), and (b) comparing standard internal calibration (circles) with modified stepwise-external calibration (squares).

different ion abundance, the error spread is probably due to a higher order effect which cannot be accounted via modified eq 3 . One possible contribution to this error is that the ion excitation is performed using a chirp waveform which may not excite all ions to the same radius. Smith and workers have demonstrated that random mass errors are reduced by using a stored waveform inverse Fourier-transform (SWIFT) excitation [44].

The tryptic digest fragments of ovalbumin $(\mathrm{m} / \mathrm{z} 1340$ to 2460) were used to verify the calibration methods. As shown in Figure 1c, eight known fragment masses are chosen for internal calibration reference points, and two noncalibrant peaks are chosen, one low $(\mathrm{m} / \mathrm{z} 2227)$ and one high $(\mathrm{m} / \mathrm{z} 2284)$ intensity peak. In Figure $5 \mathrm{a}$, the mass errors for the two tryptic peptides are individually plotted against total ion intensity for external calibration and internal calibration using standard eq 2. The mass error of $\mathrm{m} / \mathrm{z} 2227$ (open triangles) is noticeably larger than the error of $\mathrm{m} / \mathrm{z} 2284$ (filled triangles) at any given total ion intensity. This result is consistent with local-space charge effects, where $\mathrm{m} / \mathrm{z} 2227$ (open triangles) is the lower abundance ion and experiences a stronger space-charge effect. The mass errors for the two peptides are reduced using internal calibration via the standard equation (open and filled circles in Figure 5a). The global space-charge effects are largely eliminated, as the errors for the two peptides center around $0 \mathrm{ppm}$. However, the spread of the errors between the two peptides is not reduced. In Figure $5 b$, the mass errors of the two peptides are plotted against total ion intensity for standard internal calibration (open and filled circles) and modified stepwise-external calibration (open and filled squares). Although both calibration equations are able to center the errors at $0 \mathrm{ppm}$, the mass error difference between the two peptides is reduced using modified eq 3 (open and filled squares). Therefore, modified eq 3 reduces the error spread within each spectrum, an effect which is also observed for BSALow and BSAHigh in Figures $3 b$ and $4 b$.

\section{Errors for All Peaks}

For all 609 known peaks of BSALow, BSAHigh, and ovalbumin measured using a $1.0 \mathrm{~V}$ trapping potential, the RMS error is highest for external calibration, having a value of $3.4 \mathrm{ppm}$, whereas the RMS values for internal, modified internal, and modified stepwiseexternal calibration methods are $1.2 \mathrm{ppm}, 0.9 \mathrm{ppm}$, and $0.9 \mathrm{ppm}$, respectively. The mass accuracy is not limited by the small error resulting from using pseudocalibrants, i.e., masses determined by external calibration in the low trapping potential mass spectrum rather than by calculation from knowledge of their elemental composition. The pseudocalibrants are measured with an average accuracy of 0.2 to $0.3 \mathrm{ppm}$ (see Figure 2), considerably smaller than the error obtained after stepwise calibration of the mass spectrum obtained at high trapping potential (ca. $1 \mathrm{ppm}$ ). The stepwise-external calibration approach improves mass accuracy compared to conventional external calibration and provides comparable mass accuracy to internal calibration. The error distribution of stepwise-external calibration measurement is shown in Figure 6 using a 0.5 ppm bin size. The data closely resembles a Gaussian distribution (dashed line) with a small average offset of $0.14 \mathrm{ppm}$, because the reference masses are not exact, but are measured values obtained from an externally calibrated mass spectrum. The RMS and the sample standard

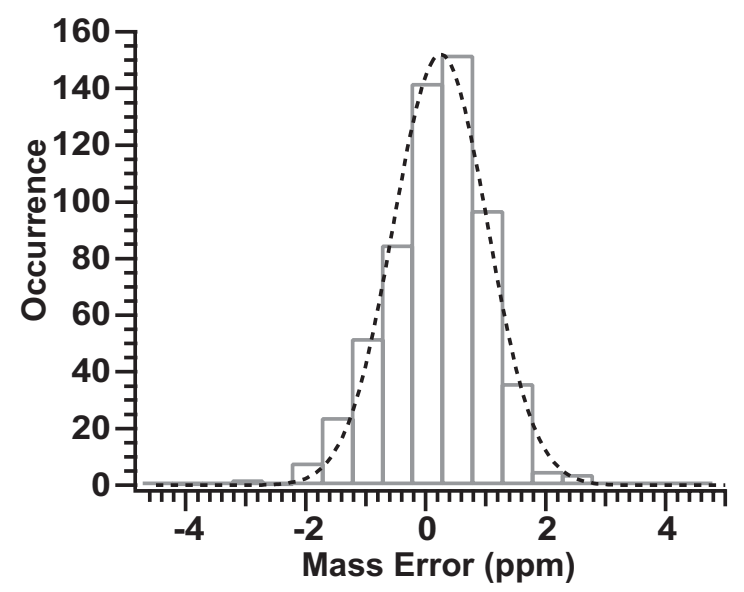

Figure 6. Histogram of mass measurement error. The 609 mass values measured from BSALow, BSAHigh, and ovalbumin experiments using stepwise-external calibration are plotted. The dashed line corresponds to a Gaussian distribution fitting of the histogram data. 
deviation values are $0.86 \mathrm{ppm}$ and $0.85 \mathrm{ppm}$, respectively. Strictly speaking, the Gaussian estimation of confidence limit is only appropriate in the absence of systematic error and, therefore, cannot be guaranteed in external calibration experiments. Nevertheless, our data absence have shown that the AVE error is a very small portion of the RMS error. Using the Gaussian distribution as a model, $99.7 \%$ of the absolute errors are estimated to be $\leq 2.6 \mathrm{ppm}$ (three times the sample standard deviation). From the actual data, only four peaks out of the total 609 peaks have mass error $>2.6$ ppm using stepwise-external calibration, corresponding to $99.3 \%$ of the errors lying within $2.6 \mathrm{ppm}$ of the true value, close to the expected value of $99.7 \%$ for a true Gaussian distribution.

\section{Advantages of Stepwise-External Calibration}

A significant advantage of internal calibration versus external calibration is that mass accuracy can be estimated for an individual spectrum, a feature which is also inherited by stepwise-external calibration. Although the mass errors of noncalibrant peaks are generally larger than those of the calibrant peaks, a strong correlation exists between the two sets of errors. For instance, spectra with higher RMS error for the calibrant peaks in Figure 2a also display higher RMS error for the noncalibrant peaks in Figure $2 \mathrm{~d}$. Therefore, the mass accuracy for internal and stepwise-external calibration experiments can be estimated on an individual spectrum basis, whereas the mass confidence in an external calibration experiment is usually estimated based on the largest errors from an ensemble of mass spectra. Consequently, the mass confidence of external calibration is always lower than that of internal and stepwiseexternal calibration.

Stepwise-external calibration avoids many challenges encountered in internal calibration experiments, such as ion suppression and spectral complexity introduced by the calibrant. For the 189 noncalibrant masses, the RMS errors of modified internal calibration and modified stepwise-external calibration are 1.2 and $0.9 \mathrm{ppm}$, respectively. To take advantage of modified calibration eq 3 , the calibrant species must span the analyte ions in both the mass range and the intensity range, and stepwise-external calibration is able to achieve this better by providing more calibrant points. As mentioned earlier, the stepwise calibration results are slightly worse when the calibrant points are limited to be the same as the ones used for internal calibration. Conversely, the mass accuracy for internal calibration can be improved when the calibrant species spans the analyte ions in both the mass range and the intensity range. However, this posts a significant challenge for proteomic mass spectrometry because separation is essential. The internal calibrant can only be added after the separation step, for example a dual-ESI source $[45,46]$ or sequential MALDI ion accumulation [38,
47], but the "proper" amount of calibrant ions to be added to the analyte is difficult to control when the total analyte ion signal varies two orders of magnitude or higher as during a typical LC experiment [40-43]. While a complex calibrant may satisfy these requirements, it will further compete with the proteome analyte for the finite ion capacity in the analyzer cell, and will diminish the useful abundance dynamic range. Stepwise-external calibration avoids this challenge by providing the means to calibrate using only the analyte peaks.

The global regression approach using eq 4 is based on the concept that the space-charge frequency shift relationship can be obtained via a series of mass spectra having different ion intensities. Up to this point, the mass errors of the global regression method are calculated under an idealized situation, where the mass distributions of the analyte and calibrant are the same. To better understand the mass confidence of this global regression approach, the 21 BSALow spectra are calibrated using the three sets of fitting parameters obtained from the BSALow, BSAHigh, and ovalbumin spectra. Only the mass errors for $m / z$ 1480, 1568, and 1640 ions are examined because these masses are covered within the three calibration ranges of BSALow, BSAHigh, and ovalbumin. The RMS error of the 21 BSALow spectra is $1.0 \mathrm{ppm}$ when calibrated based on the BSALow fitting parameters, and increases to 1.5 and $2.9 \mathrm{ppm}$ when using the BSAHigh and ovalbumin fitting parameters, respectively. The mass accuracy obtained by using the global regression method is highly dependent on the similarity between mass distributions of the analyte and the calibrant spectra and, therefore, impossible to estimate for all cases. The realistic mass error will certainly be greater than those shown in Figure 3. For comparison, a similar test is performed for stepwise-external calibration using BSALow, where the reference mass spectrum acquired at the low trapping potential is calibrated based on another BSALow spectrum, a BSAHigh spectrum, and an ovalbumin spectrum. The three resulting BSALow reference mass lists are essentially the same. The same RMS error $(0.78$ ppm) is obtained for the 21 spectra of BSALow using any of the three reference mass lists. In fact, the BSALow reference mass values acquired from the BSAHigh and ovalbumin spectra are extrapolated outside of their calibrant ranges (Figure 1), demonstrating that data extrapolation is more reliable using a low trapping potential and that the calibrant spectrum need not to have the same $m / z$ distribution as the analyte spectrum. Stepwise-external calibration is able to avoid many difficulties associated with calibrating analyte spectra of very different mass distributions because the spectra are measured under near-ideal conditions in the first step using a low trapping potential. In short, the fitting parameters $B$ and $C$ are minimized for eq 3 . The stepwise-external calibration method is developed for complex mixtures, like proteomes, where many difficulties are magnified for conventional external calibra- 
tion and conventional internal calibration approaches. However, the advantages of this method decrease when applied to less complicated samples. In the limit of studying a single compound sample, the stepwiseexternal calibration method offers no advantage.

\section{Dynamic Range}

The highest abundance peaks, measured at 0.63 and 1.0 $\mathrm{V}$ trapping potential, are used for assessing the detection dynamic range improvement for the BSALow, BSAHigh, and ovalbumin experiments. These show an average increase in dynamic range by factors of 13,5 , and 9, respectively. The dynamic range improvement for BSALow is approximately the same as the ratio of the total ion signal for the two trapping potential settings in Figure 2 (data not shown for BSAHigh and ovalbumin). Although stepwise-external calibration doubles data acquisition time, there is a vast improvement in the data that compensates for the extra effort. For a given level of mass accuracy, the abundance dynamic range of usable mass spectra increases. Taking BSALow and BSAHigh data as examples, if a RMS error limit of $<2.0 \mathrm{ppm}$ is required, then only data with total intensity less than 50 arbitrary counts are reliable using external calibration (triangles in Figures $2 \mathrm{~d}$ and $4 \mathrm{a}$ ), whereas data with intensity value within 250 arbitrary counts are reliable for the stepwise-external calibration approach (squares in Figures $3 a$ and $4 b$ ). This effectively improves the dynamic range of usable spectra by a factor of five. The dynamic range of total ion intensity among all spectra is best estimated using the lowest total ion intensity in the low trapping potential experiment to the highest total ion intensity in the high trapping potential experiment. The estimated total ion abundance dynamic ranges are 25, 19, and 40 for BSALow, BSAHigh, and ovalbumin experiments, respectively (data not shown). Although this dynamic range is lower than the typical 100 to 1000 range reported in shotgun proteomic experiments, it is important to point out that mass error is also affected by the maximum ion population. The high trapping potential used $(1.0 \mathrm{~V})$ is representative for a typical experiment and therefore the ion signal in this study is representative for the maximum total ion abundance in a typical experiment. As such, a greater abundance dynamic range can only be achieved by lowering the total ion population in the lowest abundance spectrum, and the difference in the space-charge frequency shift will be minimal. For example, the space-charge frequency shift between mass spectra of total ion signal of 30 and 1 (dynamic range of 30), is expected to be similar to that between spectra of total ion signal of 30 to 0.1 (dynamic range of 300). The additional 10-fold increase in dynamic range will only increase the frequency shift by an additional of $3 \%$ (using the first-order space charge approximation).

\section{Conclusions}

Examples of high mass accuracy by FTICR-MS can be found in the literatures [26, 28, 44, 51, 54], but these are often obtained using experimental conditions that are not optimal for high abundance dynamic range or high sensitivity. To advance the application of FT-ICR mass spectrometry for high-throughput proteomics, it is important to define procedures that achieve high mass accuracy on a routine basis. Stepwise-external calibration is a simple procedure that does not require special software or hardware, and can be adapted with any calibration equation. In stepwise-external calibration, accurate mass measurement is achieved in the first step by using a low trapping potential (high mass accuracy mode), albeit under conditions that give suboptimal sensitivity and reduced abundance dynamic range. The signal-to-noise and mass distribution are recovered in a second step by using a higher trapping potential (high abundance dynamic range mode). Of course, the mass accuracy that is obtained by using stepwise-external calibration will depend on experimental conditions, and will vary for different instruments, different samples, and different calibration equations. The data presented here are for ions with mass-to-charge values less than $\mathrm{m} / \mathrm{z} 2500$, typical of peptides from a tryptic digest. Larger mass errors may result for higher $\mathrm{m} / \mathrm{z}$ ions. Although we have only demonstrated advantages of the stepwise-external calibration for FT-ICR mass spectrometry in this paper, the approach should be applicable to other mass spectrometry methods, especially quadrupole ion trap and orbitrap mass spectrometry, where space-charge effects also limit mass accuracy.

\section{Acknowledgments}

The authors wish to thank the members of Dr. Richard D. Smith's research group, especially Dr. Joshua N. Adkins and Dr. Jason S. Page, for helpful discussions on practical mass accuracy using automatic gain control. The authors are also grateful for financial support through the National Science Foundation (grant CHE0316002) and National Institutes of Health (grant R01-RR 019767).

\section{References}

1. Yamashita, M.; Fenn, J. B. Electrospray Ion Source-Another Variation on the Free-Jet Theme. J. Phys. Chem. 1984, 88, 4451-4459.

2. Yamashita, M.; Fenn, J. B. Negative-Ion Production with the Electrospray Ion Source. J. Phys. Chem. 1984, 88, 4671-4679.

3. Fenn, J. B.; Mann, M.; Meng, C. K.; Wong, S. F.; Whitehouse, C. M. Electrospray Ionization for Mass Spectrometry of Large Biomolecules. Science 1989, 246, 64-71.

4. Karas, M.; Hillenkamp, F. Laser Desorption Ionization of Proteins with Molecular Masses Exceeding 10,000 Daltons. Anal. Chem. 1988, 60, 2299-2301.

5. Tanaka, K.; Waki, H.; Ido, Y.; Akita, S.; Yoshida, Y.; T, Y. Protein and Polymer Analyses up to $\mathrm{m} / \mathrm{z} 100,000$ by Laser Ionization Time-of-Flight Mass Spectrometry. Rapid Commun. Mass Spectrom. 1988, 2, 151-153.

6. Hillenkamp, F.; Karas, M.; Beavis, R. C.; Chait, B. T. Matrix-Assisted Laser Desorption Ionization Mass-Spectrometry of Biopolymers. Anal. Chem. 1991, 63, A1193-A1202.

7. Ge, Y.; Lawhorn, B. G.; El-Naggar, M.; Strauss, E.; Park, J. H.; Begley, T. P.; McLafferty, F. W. Top Down Characterization of Larger Proteins (45 kDa) by Electron Capture Dissociation Mass Spectrometry. J. Am. Chem. Soc. 2002, 124, 672-678.

8. Meng, F. Y.; Du, Y.; Miller, L. M.; Patrie, S. M.; Robinson, D. E.; Kelleher, N. L. Molecular-Level Description of Proteins from Saccharomyces 
cerevisiae Using Quadrupole FT Hybrid Mass Spectrometry for TopDown Proteomics. Anal. Chem. 2004, 76, 282-2858.

9. Kelleher, N. L. Top-Down Proteomics. Anal. Chem. 2004, 76, 196A-203A

10. Yates, J. R.; McCormack, A. L.; Eng, J. Mining Genomes with MS. Anal. Chem. 1996, 68, A534-A540.

11. Gygi, S. P.; Rist, B.; Gerber, S. A.; Turecek, F.; Gelb, M. H.; Aebersold, R. Quantitative Analysis of Complex Protein Mixtures Using IsotopeCoded Affinity Tags. Nat. Biotechnol. 1999, 17, 994-999.

12. Gao, H. Y.; Shen, Y. F.; Veenstra, T. D.; Harkewicz, R.; Anderson, G. A Bruce, J. E.; Pasa-Tolic, L.; Smith, R. D. Two-Dimensional Electrophoretic/ Chromatographic Separations Combined with Electrospray Ionization FTICR Mass Spectrometry for High Throughput Proteome Analysis. Microcolumn Sep. 2000, 12, 383-390.

13. Brock, A.; Horn, D. M.; Peters, E. C.; Shaw, C. M.; Ericson, C.; Phung, Q. T.; Salomon, A. R. An Automated Matrix-Assisted Laser Desorption/ Ionization Quadrupole Fourier Transform Ion Cyclotron Resonance Mass Spectrometer for "Bottom-Up" Proteomics. Anal. Chem. 2003, 75, 3419-3428.

14. Wolters, D. A.; Washburn, M. P.; Yates, J. R. An Automated Multidimensional Protein Identification Technology for Shotgun Proteomics. Anal. Chem. 2001, 73, 5683-5690.

15. Smith, R. D.; Anderson, G. A.; Lipton, M. S.; Pasa-Tolic, L.; Shen, Y. F.; Conrads, T. P.; Veenstra, T. D.; Udseth, H. R. An Accurate Mass Tag Strategy for Quantitative and High-Throughput Proteome Measurements. Proteomics 2002, 2, 513-523.

16. McDonald, W. H.; Yates, J. R. Shotgun Proteomics and Biomarker Discovery. Disease Markers 2002, 18, 99-105.

17. Washburn, M. P.; Ulaszek, R. R.; Yates, J. R. Reproducibility of Quantitative Proteomic Analyses of Complex Biological Mixtures by Multidimensional Protein Identification Technology. Anal. Chem. 2003, 75, 5054-5061.

18. Dieguez-Acuna, F. J.; Gerber, S. A.; Kodama, S.; Elias, J. E.; Beausoleil, S. A.; Faustman, D.; Gygi, S. P. Characterization of Mouse Spleen Cells by Subtractive Proteomics. Mol. Cell. Proteom. 2005, 4, 1459-1470.

19. Wong, R. L.; Amster, I. J. Combining Low and High Mass Ion Accumulation for Enhancing Shotgun Proteome Analysis by Accurate Mass Measurement. J. Am. Soc. Mass Spectrom. 2006, 17, 205-212.

20. Henzel, W. J.; Billeci, T. M.; Stults, J. T.; Wong, S. C.; Grimley, C.; Watanabe, C. Identifying Proteins from 2-Dimensional Gels by Molecular Mass Searching of Peptide-Fragments in Protein-Sequence Databases. Proc. Natl. Acad. Sci. U.S.A. 1993, 90, 5011-5015.

21. Henzel, W. J.; Watanabe, C.; Stults, J. T. Protein Identification: The Origins of Peptide Mass Fingerprinting. J. Am. Soc. Mass Spectrom. 2003, 14, 931-942.

22. Futcher, B.; Latter, G. I.; Monardo, P.; McLaughlin, C. S.; Garrels, J. I. A Sampling of the Yeast Proteome. Mol. Cell. Biol. 1999, 19, 7357-7368.

23. Krause, E.; Wenschuh, H.; Jungblut, P. R. The Dominance of ArginineContaining Peptides in MALDI-Derived Tryptic Mass Fingerprints of Proteins. Anal. Chem. 1999, 71, 4160-4165.

24. Comisarow, M. B.; Marshall, A. G. Fourier-Transform Ion-Cyclotron Resonance Spectroscopy. Chem. Phys. Lett. 1974, 25, 282-283.

25. Comisarow, M. B.; Marshall, A. G. Frequency-Sweep Fourier-Transform Ion-Cyclotron Resonance Spectroscopy. Chem. Phys. Lett. 1974, 26, 489-490.

26. Gorshkov, M. V.; Guan, S. H.; Marshall, A. G. Masses of Stable Neon Isotopes Determined at Parts-Per-Billion Precision by Fourier-Transform Ion-Cyclotron Resonance Mass-Spectrometry. Int. J. Mass Spectrom. Ion Processes 1993, 128, 47-60.

27. Rodgers, R. P.; White, F. M.; Hendrickson, C. L.; Marshall, A. G.; Andersen, K. V. Resolution, Elemental Composition, and Simultaneous Monitoring by Fourier Transform Ion Cyclotron Resonance Mass Spectrometry of Organosulfur Species Before and After Diesel Fuel Processing. Anal. Chem. 1998, 70, 4743-4750.

28. He, F.; Hendrickson, C. L.; Marshall, A. G. Baseline Mass Resolution of Peptide Isobars: A Record for Molecular Mass Resolution. Anal. Chem. 2001, 73, 647-650.

29. Clauser, K. R.; Baker, P.; Burlingame, A. L. Role of Accurate Mass Measurement $( \pm 10 \mathrm{ppm})$ in Protein Identification Strategies Employing MS or MS/MS and Database Searching. Anal. Chem. 1999, 71, 2871-2882.

30. Conrads, T. P.; Anderson, G. A.; Veenstra, T. D.; Pasa-Tolic, L.; Smith, R. D. Utility of Accurate Mass Tags for Proteome-Wide Protein Identification. Anal. Chem. 2000, 72, 3349-3354.

31. Burton, R. D.; Matuszak, K. P.; Watson, C. H.; Eyler, J. R. Exact Mass Measurements Using a 7 Tesla Fourier Transform Ion Cyclotron Resonance Mass Spectrometer in a Good Laboratory Practices-Regulated Environment. J. Am. Soc. Mass Spectrom. 1999, 10, 1291-1297.

32. Jeffries, J. B.; Barlow, S. E.; Dunn, G. H. Theory of Space-Charge Shift of Ion-Cyclotron Resonance Frequencies. Int. J. Mass Spectrom. Ion Processes 1983, 54, 169-187.

33. Francl, T. J.; Sherman, M. G.; Hunter, R. L.; Locke, M. J.; Bowers, W. D.; McIver, R. T. Experimental Determination of the Effects of Space Charge on Ion Cyclotron Resonance Frequencies. Int. J. Mass Spectrom. Ion Processes 1983, 54, 189-199.

34. Ledford, E. B.; Rempel, D. L.; Gross, M. L. Space-Charge Effects in Fourier-Transform Mass-Spectrometry-Mass Calibration. Anal. Chem. 1984, 56, 2744-2748.
35. Chen, S. P.; Comisarow, M. B. Simple Physical Models for CoulombInduced Frequency-Shifts and Coulomb-Induced Inhomogeneous Broadening for Like and Unlike Ions in Fourier-Transform IonCyclotron Resonance Mass Spectrometry. Rapid Commun. Mass Spectrom. 1991, 5, 450-455.

36. Chen, S. P.; Comisarow, M. B. Modeling Coulomb Effects in FourierTransform Ion-Cyclotron Resonance Mass-Spectrometry by Charged Disks and Charged Cylinders. Rapid Commun. Mass Spectrom. 1992, 6, $1-3$.

37. Easterling, M. L.; Mize, T. H.; Amster, I. J. Routine Part-Per-Million Mass Accuracy for High-Mass Ions: Space-Charge Effects in MALDI FT-ICR. Anal. Chem. 1999, 71, 624-632.

38. Mize, T. H.; Amster, I. J. Broad-Band Ion Accumulation with an Internal Source MALDI-FTICR-MS. Anal. Chem. 2000, 72, 5886-5891.

39. Taylor, P. K.; Amster, I. J. Space Charge Effects on Mass Accuracy for Multiply Charged Ions in ESI-FTICR. Int. J. Mass Spectrom. 2003, 222, 351-361.

40. Shen, Y. F.; Zhao, R.; Belov, M. E.; Conrads, T. P.; Anderson, G. A.; Tang, K. Q.; Pasa-Tolic, L.; Veenstra, T. D.; Lipton, M. S.; Udseth, H. R.; Smith, R. D. Packed Capillary Reversed-Phase Liquid Chromatography with High-Performance Electrospray Ionization Fourier Transform Ion Cyclotron Resonance Mass Spectrometry for Proteomics. Anal. Chem. 2001, $73,1766-1775$

41. Conrads, T. P.; Alving, K.; Veenstra, T. D.; Belov, M. E.; Anderson, G. A.; Anderson, D. J.; Lipton, M. S.; Pasa-Tolic, L.; Udseth, H. R.; Chrisler, W. B.; Thrall, B. D.; Smith, R. D. Quantitative Analysis of Bacterial and Mammalian Proteomes Using a Combination of Cysteine Affinity Tags and N-15-Metabolic Labeling. Anal. Chem. 2001, 73, 2132-2139.

42. Shen, Y. F.; Tolic, N.; Zhao, R.; Pasa-Tolic, L.; Li, L. J.; Berger, S. J.; Harkewicz, R.; Anderson, G. A.; Belov, M. E.; Smith, R. D. HighThroughput Proteomics Using High Efficiency Multiple-Capillary Liquid Chromatography with On-Line High-Performance ESI FTICR Mass Spectrometry. Anal. Chem. 2001, 73, 3011-3021.

43. Shen, Y. F.; Jacobs, J. M.; Camp, D. G.; Fang, R. H.; Moore, R. J.; Smith, R. D.; Xiao, W. Z.; Davis, R. W.; Tompkins, R. G. Ultra-High-Efficiency Strong Cation Exchange LC/RPLC/MS/MS for High Dynamic Range Characterization of the Human Plasma Proteome. Anal. Chem. 2004, 76, 1134-1144.

44. Masselon, C.: Tolmachev, A. V.; Anderson, G. A.; Harkewicz, R.; Smith, R. D. Mass Measurement Errors Caused by "Local" Frequency Perturbations in FTICR Mass Spectrometry. J. Am. Soc. Mass Spectrom. 2002, 13, 99-106.

45. Hannis, J. C.: Muddiman, D. C. A Dual Electrospray Ionization Source Combined with Hexapole Accumulation to Achieve High Mass Accuracy of Biopolymers in Fourier Transform Ion Cyclotron Resonance Mass Spectrometry. J. Am. Soc. Mass Spectrom. 2000, 11, 876-883.

46. Belov, M. E.; Zhang, R.; Strittmatter, E. F.; Prior, D. C.; Tang, K.; Smith, R. D. Automated Gain Control and Internal Calibration with External Ion Accumulation Capillary Liquid Chromatography-Electrospray Ionization-Fourier Transform Ion Cyclotron Resonance. Anal. Chem. 2003, 75, 4195-4205.

47. O'Connor, P. B.; Costello, C. E. Internal Calibration on Adjacent Samples (InCAS) with Fourier Transform Mass Spectrometry. Anal. Chem. 2000, 72, 5881-5885.

48. Muddiman, D. C. Oberg A. L. Statistical Evaluation of Internal and External Mass Calibration Laws Utilized in Fourier Transform Ion Cyclotron Resonance Mass Spectrometry. Anal. Chem. 2005, 77, $2406-$ 2414.

49. Syka, J. E. P. Marto, J. A. Bai, D. L. Horning, S. Senko, M. W. Schwartz, J. C.; Ueberheide, B.; Garcia, B.; Busby, S.; Muratore, T.; Shabanowitz, J.; Hunt, D. F. Novel Linear Quadrupole Ion Trap/FT Mass Spectrometer: Performance Characterization and Use in the Comparative Analysis of Histone H3 Post-Translational Modifications. I. Proteome Res. 2004, 3, 621-626.

50. Peterman, S. M.; Mulholland, J. J. A Novel Approach for Identification and Characterization of Glycoproteins Using a Hybrid Linear Ion Trap/FT-ICR Mass Spectrometer. J. Am. Soc. Mass Spectrom. 2006, 17, $168-179$.

51. Page, J. S.; Bogdanov, B.; Vilkov, A. N.; Prior, D. C.; Buschbach, M. A.; Tang, K.; Smith, R. D. Automatic Gain Control in Mass Spectrometry Using a Jet Disrupter Electrode in an Electrodynamic Ion Funnel. J. Am. Soc. Mass Spectrom. 2005, 16, 244-253.

52. Ramanathan, R. Introductory Econometrics with Applications; 3rd ed.; Harcourt Brace and Co.: Fort Worth, TX, 1995, p 23.

53. Shi, S. D. H.; Drader, J. J.; Freitas, M. A.; Hendrickson, C. L.; Marshall, A. G. Comparison and Interconversion of the Two Most Common Frequency-to-Mass Calibration Functions for Fourier Transform Ion Cyclotron Resonance Mass Spectrometry. Int. J. Mass Spectrom. 2000, 196, 591-598.

54. Gorshkov, M. V.; Marshall, A. G.; Nikolaev, E. N. Analysis and Elimination of Systematic-Errors Originating from Coulomb Mutual Interaction and Image Charge in Fourier-Transform Ion-Cyclotron Resonance Precise Mass Difference Measurements. J. Am. Soc. Mass Spectrom. 1993, 4, 855-868. 